\title{
Tuberculosis: efectividad de una estrategia para mejorar la adherencia al tratamiento
}

Effectiveness of a strategy to improve adherence to tuberculosis treatment

Thiam S, et al. JAMA 2007; 297:380-386

\section{Objetivo}

Evaluar la efectividad de una estrategia de tratamiento supervisado (en inglés DOTS) y su potencial impacto en el control de la tuberculosis en países de bajos recursos.

\section{Diseño}

Ensayo clínico controlado aleatorizado (ECCA) por "clusters"*.

\section{Lugar}

Centros de salud gubernamentales en Senegal.

\section{Pacientes}

Mayores de 15 años con tuberculosis pulmonar con baciloscopía positiva recientemente diagnosticada atendidos en 16 diferentes centros de salud.

\section{Intervención}

Estrategias de comunicación y consejo a pacientes, descentralización de los tratamientos a los puestos sanitarios locales, elección del supervisor del tratamiento por parte de los pacientes y coordinación de actividades de supervisión del programa. El grupo control fue seguido bajo el programa de control de tuberculosis vigente en Senegal.

\section{Medición de resultados principales}

Proporción de pacientes que se curaron y/o finalización completamente el tratamiento completo. También se estimó la proporción de pacientes que abandonaron el tratamiento en cada grupo.

\section{Resultados principales}

Ver tabla 1.

Tabla 1: comparación de las diferentes estrategias de tratamiento antituberculoso.

\begin{tabular}{|c|c|c|c|c|c|}
\hline & $\begin{array}{c}\text { Supervisado } \\
\mathbf{n}=778\end{array}$ & $\begin{array}{c}\text { Control } \\
n=44\end{array}$ & RR* (IC95\%) $^{*}$ & $\begin{array}{l}\text { Beneficio } \\
\text { atribuible }\end{array}$ & NNT \\
\hline Exito $^{a}$ & $88 \%$ & $76 \%$ & $1,18(1,03$ a 1,34$)$ & $12 \%$ & 8 \\
\hline Cura & $83,4 \%$ & $70 \%$ & $1,19(1,03$ a 1,35$)$ & $13,4 \%$ & 8 \\
\hline Abandono & $5,5 \%$ & $16,8 \%$ & $0,43(0,21$ a 0,89$)$ & $11 \%$ & 9 \\
\hline Falla & $12 \%$ & $24 \%$ & $0,5(0,34$ a 0,78$)$ & $12 \%$ & 8 \\
\hline
\end{tabular}

á́xito: suma de pacientes con cura documentada y de pacientes que completaron los seis meses de tratamiento pero no hicieron el esputo de control.

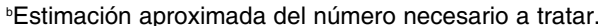

\section{Conclusiones}

Una intervención que combinó entrenamiento del personal, elección del supervisor y refuerzo de las actividades de control del programa mejoró los resultados alcanzados por los pacientes. Esta estrategia podría ser generalizada con éxito a otros países de escasos recursos.

Palabras Claves: control de la tuberculosis, terapia supervisada. Key Words: tuberculosis control, directly observed therapy. Fuente de financiamiento: Ministerio de Investigación de Francia.
Hace un año discutimos una revisión Cochrane sobre la efectividad de la estrategia DOTS para el control y tratamiento de la tuberculosis ${ }^{1,2}$. Las distintas visiones recomendaban implementar y evaluar estrategias para mejorar la adherencia de los pacientes y la conclusión argumentaba que el componente de supervisión directa del tratamiento no había demostrado eficacia en ECCA y que, en consecuencia, el costo de su implementación no era justificable, especialmente en países de bajos recursos.

Cuando se pretende evaluar el impacto de estrategias comunitarias no es sencillo desagregar los efectos de uno solo de sus componentes, y es cierto que la recomendación universal de la estrategia DOTS no ha incluido guías para la provisión de cada uno de ellos, por lo que su impacto se ha limitado por las dificultades propias de su implementación en cada región ${ }^{2,3}$. A partir de los resultados de las investigaciones operativas se deben desarrollar programas sensibles al contexto local en los diferentes niveles (pacientes y sus familias, sistema de salud y comunidad) para lograr el impacto esperado ${ }^{3}$. Este excelente ECCA comunitario demuestra un beneficio modesto de una estrategia adaptada al contexto local, destacándose que la supervisión directa fue asumida en el grupo intervención, mayormente (60\%) por fami- liares; lo que coincide con lo reportado por Kamolratanakul ${ }^{4}$, en el único ECCA incluido en la revisión Cochrane que había demostrado beneficios similares. Los autores fueron conservadores en el reporte de los resultados, ya que si hubieran considerado la falla del tratamiento como el evento primario de interés, la estrategia habría presentado una reducción relativa del $50 \%$ en la tasa de falla en lugar de un $R R=1,18$ en la tasa de éxito, haciendo más impactante el resultado con los mismos hallazgos.

\section{Conclusiones del comentador}

El impacto de la tuberculosis en regiones pobres es sustancial desde lo epidemiológico y lo económico. Los estudios de costo efectividad brindan un marco explícito para asistir la toma de decisiones. La estrategia DOTS demostró ser altamente costoefectiva en países con alta carga de enfermedad y se debería recomendar como prioritaria ${ }^{5}$. En los de carga intermedia como nuestra región, en los que existen programas de control de efectividad variable, será importante evaluar el costo y la efectividad incremental $^{\star}$ de implementarla al menos en las áreas de mayor impacto.

Fernando Rubisntein [ Servicio de Medicina Familiar y Comunitaria del Hospital Italiano de Buenos Aires. fernando.rubintein@hospitalitaliano.org.ar ]

Recibido el 20/01/08 y aceptado el 11/02/08.

Rubinstein F.Tuberculosis: Efectividad de una estrategia para mejorar la adherencia al tratamiento. Evid actual pract ambul 11(2); 40 Mar-Abr. Thiam $\mathrm{S}$, et al. Effectiveness of a Strategy to Improve adherence to Tuberculosis treatment in a resource poor setting. A cluster randomized Controlled trial. JAMA 2007; 297:380-386. PMID:17244834.

Referencia

1. Simmons M, Rubinstein F. Controversia sobre la efectividad del tratamiento supervisado en tuberculosis. Evid. actual. práct. ambul; 10(1): 23-25, ene-feb.2007.

2. Volmink J, Garner P. Directly observed therapy for treating tuberculosis. Cochrane Database of Systematic Reviews 2007, Issue 4. Art. No.: CD003343. DOI: 10.1002/14651858.CD003343.pub3.

3. Lienhardt C, Ogden JA. Tuberculosis control in resource-poor countries: have we reached the limits of the universal paradigm? Trop Med Int Health. 2004 Jul;9(7):833-41. 4. Kamolratanakul P, Sawert $\mathrm{H}$ et al. Randomized controlled trial of directly observed treatment (DOT) for patients with pulmonary tuberculosis in Thailand. Trans $\mathrm{R}$ Soc Trop Med Hyg. 1999 Sep-Oct:93(5):552-7.

5. Baltussen, R, Floyd, $\mathrm{K}$ et al. Cost effectiveness analysis of strategies for tuberculosis control in developing countries. BMJ 2005;331:1364 (10 December), doi:10.1136/bmj.38645.660093.68 (published 10 November 2005) 